\title{
A mean-variance control framework for platoon control problems: Weak convergence results and applications on reduction of complexity
}

\author{
Zhixin Yang, G. Yin, Le Yi Wang and Hongwei Zhang \\ Dedicated to Wing Shing Wong on the occasion of his 60th Birthday
}

\begin{abstract}
This paper introduces a new approach of treating platoon systems using mean-variance control formulation. The underlying system is a controlled switching diffusion in which the random switching process is a continuous-time Markov chain. This switching process is used to represent random environment and other random factors that cannot be represented by stochastic differential equations driven by a Brownian motion. The state space of the Markov chain is large in our setup, which renders practically infeasible for a straightforward implementation of the mean-variance control strategy obtained in the literature. By partitioning the states of the Markov chain into sub-groups (or clusters) and then aggregating the states of each cluster as a super state, we are able to obtain a limit system of much reduced complexity. The justification of the limit system is rigorously supported by establishing certain weak convergence results.
\end{abstract}

KEYWORds AND PHRASEs: platoon control, mean-variance control, two-time-scale model, weak convergence, reduction of complexity.

\section{Introduction}

Highway vehicle control is a critical task in developing intelligent transportation systems. Platoon formation has been identified as one promising

This research was supported in part by the National Science Foundation under CNS-1136007. 
strategy for enhanced safety, improved highway utility, increased fuel economy, and reduced emission toward autonomous or semi-autonomous vehicle control. The goal of longitudinal platoon control is to ensure that all the vehicles move in the same lane at the same speed with desired inter-vehicle distances.

Platoon control has been studied in the contexts of intelligent highway control and automated highway systems for many years with numerous methodologies and demonstration systems [3, 8]. Many control methodologies have been applied, including PID controllers, state feedback, adaptive control, state observers, among others, with safety, string stability, and team coordination as the most common objectives [1, 6, 11].

A platoon may be viewed as a networked system consisting of individual subsystems whose operations and resource consumptions must be carefully coordinated to achieve desired performance for the entire platoon. Platoon control and performance optimization bear certain similarity to portfolio management in mathematical finance in which optimal distribution of available resources to different subsystems (stocks or mutual funds) can lead to increased return and reduced risk. By borrowing the basic idea of meanvariance control from mathematical finance, we study its potential extension and applications to platoon control systems.

The origin of the mean-variance optimization problem can be traced back to the Nobel-prize-winning work of Markowitz [7. The salient feature of the model is that, in the context of finance, it enables an investor to seek highest return after specifying the acceptable risk level quantified by the variance of the return. The mean-variance approach has become the foundation of modern finance theory and has inspired numerous extensions and applications. Using the stochastic linear-quadratic (LQ) control framework, Zhou and Li [17] studied the mean-variance problem for a continuous-time model. Note that the problem becomes fundamentally different from the traditional LQ problem studied in literature. In the classical time-honored LQ theory, the matrix related to the control (known as control weight) needs to be positive definite. In the mean variance setup for linear systems, the control weight is non-positive definite. In our previous work [18, the meanvariance problems for switching diffusion models were treated and a number of results including optimal portfolio selection, efficient frontier, and mutual fund theory were discovered.

In this study, we identify the following three scenarios in platoon control problems in which resource allocation and risk management lead naturally to mean-variance formulations. 
1) Consider the longitudinal inter-vehicle distance control. To increase highway utility, it is desirable to reduce the total length of a platoon, which intends to reduce inter-vehicle distances. This strategy, however, will increase the risk of collision in the presence of vehicle traffic uncertainties. This tradeoff amounts to maximizing benefits at a tolerable risk. This may be compared to financial portfolio management problems in which one wants to maximize profit return but control the risk too. Consequently, the basic idea of mean-variance (MV) control becomes useful. The MV approach has never been applied to platoon control. It offers several distinct advantages: 1) Unlike heuristic methods such as neural network optimization and genetic algorithms, the MV method is simple but rigorous; 2) the MV method is computationally efficient; 3 ) the form of the solution (i.e., efficient frontier) is readily applicable to assessing risks in platoon formation, hence is practically appealing.

2) Consider communication resource allocation of bandwidths for vehicle to vehicle (V2V) communications. For a given maximum throughput of a platoon communication system, the communication system operator must find a way to assign this resource to different vehicle-tovehicle channels. Each channel's bandwidth usage is the state of the subsystem. Their summation is a random process and is desired to approach the maximum throughput (the desired mean at the terminal time) with small variations. Consequently, it becomes a mean-variance control problem.

3) We may view platoon fuel consumption (or similarly, total emission) in the MV setting. The platoon fuel consumption is the summation of vehicle fuel consumptions. Due to variations in vehicle sizes and speeds, each vehicle's fuel consumption is a controlled random process. Tradeoff between a platoon's team acceleration/maneuver capability and fuel consumption can be summarized as a desired platoon fuel consumption rate. Assigning allowable fuel consumption rates to different vehicles result in coordination of vehicle operations modeled by subsystem fuel rate dynamics. To control the platoon fuel consumption rate to be close to the designated value, one may formulate this as a mean-variance control problem.

Due to vehicle mobility and network resource fluctuations, platoon network topologies or random environment may vary dynamically. To capture this common feature in platoon control, we model the network topology or 
the environment as a continuous-time Markov chain. The resulting system becomes one having regime switching. We assume that the Markov chain has a large state space in order to deal with complex systems. To treat the platoon problems, we could in principle apply the results in 18 . Nevertheless, the large state space of the Markov chain renders a straightforward implementation of the mean-variance control strategy obtained in [18 practically infeasible. The computational complexity becomes a major concern. Inspired by the idea in the work [10], to exploit the hierarchical structure of the underlying systems, and to fully utilize the near decomposability [2, 9] by means of considering fast and slow switching modes, the work [16] treated near-optimal control problems of LQG with regime switching. However, only positive definite control weights were allowed there under the usual quadratic control criteria. In our current setup, the control weights are indefinite, so the main assumption in [16] does not hold. Physically, only part of the network topology or random environment will change at a time such as the addition/departure of a vehicle, or the loss/addition of a communication link. Some parts of the chain vary rapidly (e.g., when vehicles pass some bridges that block signal transmissions) and others change slowly (e.g., when vehicles are moving smoothly in open space). The fast and slow variations are in high contrast, resulting in a two-time-scale formulation. This paper, together with its companion paper [13], sets up a new formulation towards resolving platoon coordination and optimization issues with reduced computational complexity.

This two-time-scale scenario provides an opportunity to reduce computational complexity for the Markov chain. The main idea is a decomposition of the large space into sub-clusters and aggregation of states in each sub-cluster. That is, we partition the state space of the Markov chain into subspaces (or sub-groups or sub-clusters). Then, in each of the sub-clusters, we aggregate all the states into one super state. Thus the total number of discrete states is substantially reduced. In the companion paper [13, we obtained near-optimal controls by designing controls using a "limit system" and such constructed controls are nearly optimal. This paper focuses on justifying the limit system by means of weak convergence methods. The weak convergence result is proved using a martingale problem formulation. As a first step in this direction, this paper presents the key mathematical framework and core results. Their usage can be expanded by considering further system details in practical applications with concrete model structures, sizes, resource definitions, and physical limitations. These will be investigated and reported elsewhere. 
The rest of the paper is arranged as follows. Section 2 formulates the two-time-scale platoon problems. Section 3 proceeds with the study of the underlying mean-variance problem. Section 4 derives weak convergence of underlying systems using martingale problem formulation, which rigorously justifies the use of the limit system. The limit system has a substantially fewer number of discrete states, resulting in much reduced complexity. Section 5 recalls the near-optimal controls obtained in [13] and presents numerical experiments that further illustrate the near optimality. Finally, Section 6 concludes the paper with some final remarks.

\section{Problem formulation}

We work with a complete probability space $(\Omega, \mathcal{F}, P)$. Suppose that $\alpha(t)$ is continuous-time Markov chain with state space $\mathcal{M}=\{1,2, \ldots, m\}$, that $w(t)=\left(w_{1}(t), w_{2}(t), \ldots, w_{d}(t)\right)^{\prime}$ is a standard $d$-dimensional Brownian motion, where $a^{\prime}$ denotes the transpose of $a \in \mathbb{R}^{l_{1} \times l_{2}}$ with $l_{i} \geq 1$, and $\mathbb{R}^{r \times 1}$ is simply written as $\mathbb{R}^{r}$ in what follows. Suppose that $w(t)$ and the Markov chain are independent of each other. In [18], a mean-variance portfolio selection problem in which the environment is randomly varying and modeled by a regime-switching system was treated. In this paper, we continue to use the same setup as in [18. In addition to the finance applications, we are particularly interested in platoon control problems. Mathematically, the new feature considered here is that the state space of the discrete event process $\alpha(\cdot)$ is large. Obtaining the optimal strategy in such a large-scale system involves high computational complexity, optimal control a difficult task. To reduce the computational complexity, we note that in the Markov chain, some groups of states vary rapidly whereas others change slowly. Using the distinct transition rates, we decompose the state space $\mathcal{M}$ into subspaces $\mathcal{M}=\cup_{i=1}^{l} \mathcal{M}_{i}$ such that within each $\mathcal{M}_{i}$, the transitions happen frequently and among different clusters the transitions are relatively infrequent. To reflect the different transition rates, we let $\alpha(t)=\alpha^{\varepsilon}(t)$ where $\varepsilon>0$ is a small parameter so that the generator of the Markov chain is given by

$$
Q^{\varepsilon}=\frac{\widetilde{Q}}{\varepsilon}+\widehat{Q} .
$$

We consider a network that contains $d_{1}+1$ nodes. The flow of one of the nodes is given by stochastic ODE

$$
\begin{aligned}
d x_{0}^{\varepsilon}(t) & =r\left(t, \alpha^{\varepsilon}(t)\right) x_{0}^{\varepsilon}(t) d t \\
x_{0}^{\varepsilon}(0) & =x_{0}, \quad \alpha^{\varepsilon}(0)=\alpha .
\end{aligned}
$$


where for each $j \in \mathcal{M}, r(t, j) \geq 0$ is the growth rate corresponding to $x_{0}^{\varepsilon}(t)$. The flows of the other $d_{1}$ nodes follow geometric Brownian motion models under random environments (random switching)

$$
\begin{aligned}
d x_{i}^{\varepsilon}(t) & =x_{i}^{\varepsilon}(t) r_{i}\left(t, \alpha^{\varepsilon}(t)\right) d t+x_{i}^{\varepsilon}(t) \sigma_{i}\left(t, \alpha^{\varepsilon}(t)\right) d w(t) \\
x_{i}^{\varepsilon}(0) & =x_{i}, \quad \alpha_{i}^{\varepsilon}(0)=\alpha, \quad \text { for } i=1,2, \ldots, d_{1} .
\end{aligned}
$$

where

$$
\begin{aligned}
\sigma_{i}\left(t, \alpha^{\varepsilon}(t)\right) & =\left(\sigma_{i 1}\left(t, \alpha^{\varepsilon}(t)\right), \sigma_{i 2}\left(t, \alpha^{\varepsilon}(t)\right), \ldots, \sigma_{i d}\left(t, \alpha^{\varepsilon}(t)\right)\right) \in \mathbb{R}^{1 \times d} \\
w(t) & =\left(w_{1}(t), w_{2}(t), \ldots, w_{d}(t)\right)^{\prime} \in \mathbb{R}^{d \times 1}
\end{aligned}
$$

and $r_{i}(t, j) \in \mathbb{R}$ (with $i=1, \ldots, d_{1}$ and $j \in \mathcal{M}$ ) is the drift rate for the flow of the ith node. We can represent the total flows of the whole network system as a 1-dimensional variable $x^{\varepsilon}(t)$ for which we need to decide the proportion $n_{i}(t)$ of flow $x_{i}^{\varepsilon}(t)$ to put on node $i$, i.e.,

$$
x^{\varepsilon}(t)=\sum_{i=0}^{d_{1}} n_{i}(t) x_{i}^{\varepsilon}(t) .
$$

By assuming that the interaction among these $d_{1}+1$ nodes occurs continuously, we have

$$
\begin{aligned}
d x^{\varepsilon}(t) & =\sum_{i=0}^{d_{1}} n_{i}(t) d x_{i}^{\varepsilon}(t) \\
& =\left[r\left(t, \alpha^{\varepsilon}(t)\right) x^{\varepsilon}(t)+B\left(t, \alpha^{\varepsilon}(t)\right) u(t)\right] d t+u^{\prime}(t) \sigma\left(t, \alpha^{\varepsilon}(t)\right) d w(t) \\
x^{\varepsilon}(0) & =x=\sum_{i=1}^{d_{1}} n_{i}(0) x_{i}, \quad \alpha^{\varepsilon}(0)=\alpha, \quad \text { for } 0 \leq t \leq T
\end{aligned}
$$

where

$$
\begin{aligned}
B\left(t, \alpha^{\varepsilon}(t)\right)= & \left(r_{1}\left(t, \alpha^{\varepsilon}(t)\right)-r\left(t, \alpha^{\varepsilon}(t)\right), r_{2}\left(t, \alpha^{\varepsilon}(t)\right)-r\left(t, \alpha^{\varepsilon}(t)\right), \ldots,\right. \\
& \left.r_{d_{1}}\left(t, \alpha^{\varepsilon}(t)\right)-r\left(t, \alpha^{\varepsilon}(t)\right)\right), \\
\sigma\left(t, \alpha^{\varepsilon}(t)\right)= & \left(\sigma_{1}\left(t, \alpha^{\varepsilon}(t)\right), \ldots, \sigma_{d_{1}}\left(t, \alpha^{\varepsilon}(t)\right)\right)^{\prime} \in \mathbb{R}^{d_{1} \times d} \\
u(t)= & \left(u_{1}(t), \ldots, u_{d_{1}}(t)\right)^{\prime} \in \mathbb{R}^{d_{1} \times 1}
\end{aligned}
$$

and $u_{i}(t)=n_{i}(t) x_{i}(t)$ is the total amount of flow for node $i$ at time $t$ for $i=$ $1,2, \ldots, d_{1}$. We assume throughout this paper that all the functions $r(t, i)$, $B(t, i)$, and $\sigma(t, i)$ are measurable and uniformly bounded in $t$. We also 
assume the non-degeneracy condition is satisfied, i.e., there is a $\delta>0$ such that $a(t, i)=\sigma(t, i) \sigma^{\prime}(t, i) \geq \delta I$ for any $t \in[0, T]$ and $i \in \mathcal{M}$. We denote by $L_{\mathcal{F}}^{2}\left(0, T ; \mathbb{R}^{l_{0}}\right)$ the set of all $\mathbb{R}^{l_{0}}$-valued, measurable stochastic processes $f(t)$ adapted to $\left\{\mathcal{F}_{t}\right\}_{t \geq 0}$ such that $E \int_{0}^{T}|f(t)|^{2} d t<+\infty$.

Let $\mathcal{U}$ be the set of controls which is a compact set in $\mathbb{R}^{d_{1} \times 1}$. The $u(\cdot)$ is said to be admissible if $u(\cdot) \in L_{\mathcal{F}}^{2}\left(0, T ; \mathbb{R}^{d_{1}}\right)$ and the Equation 2.4 has a unique solution $x^{\varepsilon}(\cdot)$ corresponding to $u(\cdot)$. In this case, we call $\left(x^{\varepsilon}(\cdot), u(\cdot)\right)$ an admissible (total flow, flow distribution) pair. Our objective is to find an admissible control $u(\cdot)$ among all the admissible controls given that the expected terminal flow value of the whole system is $\operatorname{Ex}^{\varepsilon}(T)=z$ for some given $z \in \mathbb{R}$ so that the risk measured by the variance at the terminal of the flow is minimized. Specifically, we have the following performance measure

$$
\begin{aligned}
& \min \left\{J(x, \alpha, u(\cdot))=E\left[x^{\varepsilon}(T)-z\right]^{2}\right\} \\
& \text { subject to } E x^{\varepsilon}(T)=z .
\end{aligned}
$$

Note that in this case, the objective function does not involve control $u$. Thus, the LQG problem is one with zero control weight hence the problem becomes one with indefinite control weights.

\section{Feasibility and optimal controls}

To begin, we present the following lemma, whose proof can be found in [18, Theorem 3.3].

Lemma 3.1. The mean variance problem (2.5) is feasible for every $z \in \mathbb{R}$ if and only if

$$
E\left\{\int_{0}^{T}\left|B\left(t, \alpha^{\varepsilon}(t)\right)\right|^{2} d t\right\}>0 .
$$

To study optimality and to handle the constraint in (2.5), we apply the Lagrange multiplier technique and get unconstrained problem (see, e.g., [18]) with multiplier $\lambda \in \mathbb{R}$ :

$$
\begin{aligned}
& \min \left\{J(x, \alpha, u(\cdot), \lambda)=E\left[x^{\varepsilon}(T)+\lambda-z\right]^{2}-\lambda^{2}\right\} \\
& \text { subject to }\left(x^{\varepsilon}(\cdot), u(\cdot)\right) \text { admissible } .
\end{aligned}
$$

To find the minimum of $J(x, \alpha, u(\cdot), \lambda)$, it suffices to select $u(\cdot)$ such that $E\left(x^{\varepsilon}(T)+\lambda-z\right)^{2}$ is minimized. We regard this part as $J^{\varepsilon}(x, \alpha, u(\cdot))$ in the 
sequel. Let $v^{\varepsilon}(x, \alpha)=\inf _{u(\cdot)} J^{\varepsilon}(x, \alpha, u(\cdot))$ be the value function. First define

$$
\rho(t, i)=B(t, i)\left[\sigma(t, i) \sigma^{\prime}(t, i)\right]^{-1} B^{\prime}(t, i), \quad i \in\{1,2, \ldots, m\}
$$

Consider the following two systems of ODEs for $i=1,2, \ldots, m$ :

$$
\begin{aligned}
& \dot{P}^{\varepsilon}(t, i)=P^{\varepsilon}(t, i)[\rho(t, i)-2 r(t, i)]-\sum_{j=1}^{m} q_{i j}^{\varepsilon} P^{\varepsilon}(t, j) \\
& P^{\varepsilon}(T, i)=1 .
\end{aligned}
$$

and

$$
\begin{aligned}
\dot{H}^{\varepsilon}(t, i)= & H^{\varepsilon}(t, i) r(t, i)-\frac{1}{P^{\varepsilon}(t, i)} \sum_{j=1}^{m} q_{i j}^{\varepsilon} P^{\varepsilon}(t, j) H^{\varepsilon}(t, j) \\
& +\frac{H^{\varepsilon}(t, i)}{P^{\varepsilon}(t, i)} \sum_{j=1}^{m} q_{i j}^{\varepsilon} P^{\varepsilon}(t, j), \\
H^{\varepsilon}(T, i)= & 1 .
\end{aligned}
$$

The existence and uniqueness of solutions to the above two systems of equations are easy to obtain since they are both linear in the continuous state variable. Applying the generalized Itô's formula to

$$
v^{\varepsilon}\left(t, x^{\varepsilon}(t), i\right)=P^{\varepsilon}(t, i)\left(x^{\varepsilon}(t)+(\lambda-z) H^{\varepsilon}(t, i)\right)^{2},
$$

by employing the completing square techniques, we obtain

$$
\begin{aligned}
& d P^{\varepsilon}(t, i)\left[x^{\varepsilon}(t)+(\lambda-z) H^{\varepsilon}(t, i)\right]^{2} \\
= & 2 P^{\varepsilon}(t, i)\left[x^{\varepsilon}(t)+(\lambda-z) H^{\varepsilon}(t, i)\right] d x^{\varepsilon}(t)+P^{\varepsilon}(t, i)\left(d x^{\varepsilon}(t)\right)^{2} \\
& +\sum_{j=1}^{m} q_{i j}^{\varepsilon} P^{\varepsilon}(t, j)\left[x^{\varepsilon}(t)+(\lambda-z) H^{\varepsilon}(t, j)\right]^{2} d t \\
& +\dot{P}^{\varepsilon}(t, i)\left[x^{\varepsilon}(t)+(\lambda-z) H^{\varepsilon}(t, i)\right]^{2} d t \\
& +2 P^{\varepsilon}(t, i)\left[x^{\varepsilon}(t)+(\lambda-z) H^{\varepsilon}(t, i)\right](\lambda-z) \dot{H}^{\varepsilon}(t, i) d t .
\end{aligned}
$$


Therefore, after plugging in the dynamic equation of $P^{\varepsilon}(t, i)$ and $H^{\varepsilon}(t, i)$, integrating from 0 to $T$, and taking expectation, we obtain

$$
\begin{aligned}
& E\left[x^{\varepsilon}(T)+\lambda-z\right]^{2} \\
= & P^{\varepsilon}(0, \alpha)\left[x+(\lambda-z) H^{\varepsilon}(0, \alpha)\right]^{2} \\
& +E \int_{0}^{T}(\lambda-z)^{2} \sum_{j=1}^{m} q_{i j}^{\varepsilon} P^{\varepsilon}(t, j)\left[H^{\varepsilon}(t, j)-H^{\varepsilon}(t, i)\right]^{2} d t \\
& +E \int_{0}^{T} P^{\varepsilon}(t, i)\left(u(t)-u^{\varepsilon, *}(t)\right)^{\prime}\left(\sigma(t, i) \sigma^{\prime}(t, i)\right)\left(u(t)-u^{\varepsilon, *}(t)\right) d t .
\end{aligned}
$$

This leads to the optimal control of the form

$$
\begin{aligned}
u^{\varepsilon, *}\left(t, \alpha^{\varepsilon}(t), x^{\varepsilon}(t)\right)= & -\left(\sigma\left(t, \alpha^{\varepsilon}(t)\right) \sigma^{\prime}\left(t, \alpha^{\varepsilon}(t)\right)\right)^{-1} \\
& \cdot B^{\prime}\left(t, \alpha^{\varepsilon}(t)\right)\left[x^{\varepsilon}(t)+(\lambda-z) H^{\varepsilon}\left(t, \alpha^{\varepsilon}(t)\right)\right] .
\end{aligned}
$$

To proceed, we state a lemma below for subsequent use. The proof of the lemma is omitted.

Lemma 3.2. The following assertions hold.

- The solutions of Equations (3.3) and (3.4) satisfy $0<P^{\varepsilon}(t, i) \leq c$ and $0<H^{\varepsilon}(t, i) \leq 1$ for all $t \in[0, T], i=1,2, \ldots, m$.

- For $i \in \mathcal{M}$, the solutions of (3.3) and (3.4) are uniformly Lipschitz on $[0, T]$.

\section{Weak convergence results}

Although the optimal solution of the mean-variance control problem for the regime-switching system can be obtained using the methods developed in [18], the difficulty is that $|\mathcal{M}|=m$ is large and we have to solve a large-scale system, which is computationally intensive and practically unattractive. As a viable alternative, we focus on an decomposition-aggregation approach.

Assume that $\widetilde{Q}$ is of the block-diagonal form $\widetilde{Q}=\operatorname{diag}\left(\widetilde{Q}^{1}, \ldots, \widetilde{Q}^{l}\right)$ in which $\widetilde{Q}^{k} \in \mathbb{R}^{m_{k} \times m_{k}}$ are irreducible for $k=1,2, \ldots, l$ and $\sum_{k=1}^{l} m_{k}=m$, and $\widetilde{Q}^{k}$ denotes the $k$ th block matrix in $\widetilde{Q}$. Let $\mathcal{M}_{k}=\left\{s_{k 1}, s_{k 2}, \ldots, s_{k m_{k}}\right\}$ denote the states corresponding to $\widetilde{Q}^{k}$ and let $\mathcal{M}=\mathcal{M}_{1} \cup \mathcal{M}_{2} \ldots \cup \mathcal{M}_{l}=$ $\left\{s_{11}, s_{12}, \ldots, s_{1 m_{1}}, \ldots, s_{l 1}, s_{l 2}, \ldots, s_{l m_{l}}\right\}$. The slow and fast components are coupled through weak and strong interactions in the sense that the underlying Markov chain fluctuates rapidly within a single group $\mathcal{M}_{k}$ and jumps less frequently among groups $\mathcal{M}_{k}$ and $\mathcal{M}_{j}$ for $k \neq j$. 
By aggregating the states in $\mathcal{M}_{k}$ as one state $k$, we can obtain an aggregated process $\bar{\alpha}^{\varepsilon}(\cdot)$. That is, $\bar{\alpha}^{\varepsilon}(t)=k$ when $\alpha^{\varepsilon}(t) \in \mathcal{M}_{k}$. By virtue of [14, Theorem7.4], $\bar{\alpha}^{\epsilon}(\cdot)$ converges weakly to $\bar{\alpha}(\cdot)$ whose generator is given by

$$
\bar{Q}=\operatorname{diag}\left(\mu^{1}, \mu^{2}, \ldots, \mu^{l}\right) \widehat{Q} \operatorname{diag}\left(\mathbb{1}_{m_{1}}, \mathbb{1}_{m_{2}}, \ldots, \mathbb{1}_{m_{l}}\right),
$$

where $\mu^{k}$ is the stationary distribution of $\widetilde{Q}^{k}, k=1,2, \ldots, l$, and $\mathbb{1}_{n}=(1,1$, $\ldots, 1) \in \mathbb{R}^{n}$. Define an operator $\mathcal{L}^{\varepsilon}$ by

$$
\begin{aligned}
\mathcal{L}^{\varepsilon} f(x, t, \iota)= & \frac{\partial f(x, t, \iota)}{\partial t}+[r(t, \iota) x+B(t, \iota) u(t)] \frac{\partial f(x, t, \iota)}{\partial x} \\
& +\frac{1}{2}\left[u^{\prime}(t) \sigma(t, \iota) \sigma^{\prime}(t, \iota) u(t)\right] \frac{\partial^{2} f(x, t, \iota)}{\partial x^{2}} \\
& +Q^{\varepsilon} f(x, t, \iota), \quad \iota \in \mathcal{M},
\end{aligned}
$$

where

$$
Q^{\varepsilon} f(x, t, \cdot)(\iota)=\sum_{\ell \neq \iota} q_{\iota \ell}^{\varepsilon}(f(x, t, \ell)-f(x, t, \iota)),
$$

and for each $\iota \in \mathcal{M}, f(\cdot, \cdot, \iota) \in C^{2,1}$ (that is, $f(\cdot)$ has continuous derivatives up to the second order with respect to $x$ and continuous derivative with respect to $t$ up to the first order). Note that the operator, in fact, is $u$ dependent, so it may be written as $\mathcal{L}^{u}$. In this paper, we work with a fixed $u$. We could also consider a feedback system with $\varepsilon$-dependence in the control. In such a setup, we can use a relaxed control formulation. However, we will not proceed in this line here. Define

$$
\begin{aligned}
\overline{\mathcal{L}} f(x, t, k)= & \frac{\partial f(x, t, k)}{\partial t}+[\bar{r}(t, k) x+\bar{B}(t, k) u(t)] \frac{\partial f(x, t, k)}{\partial x} \\
& +\frac{1}{2}\left[u^{\prime}(t) \bar{\sigma}(t, k) \bar{\sigma}^{\prime}(t, k) u(t)\right] \frac{\partial^{2} f(x, t, k)}{\partial x^{2}} \\
& +\bar{Q} f(x, t, k), \quad k \in \overline{\mathcal{M}},
\end{aligned}
$$

where $\bar{Q}$ is defined in 4.1 and

$$
\begin{aligned}
\bar{r}(t, k) & =\sum_{j=1}^{m_{k}} \mu_{j}^{k} r\left(t, s_{k j}\right), \quad \bar{B}(t, k)=\sum_{j=1}^{m_{k}} \mu_{j}^{k} B\left(t, s_{k j}\right), \\
\bar{\sigma}^{2}(t, k) & =\sum_{j=1}^{m_{k}} \mu_{j}^{k} \sigma^{2}\left(t, s_{k j}\right) .
\end{aligned}
$$


The following theorems are concerned with the weak convergence of a pair of processes.

Theorem 4.1. Suppose that the martingale problem with operator $\overline{\mathcal{L}}$ defined in (4.4) has a unique solution for each initial condition. Then the pair of processes $\left(x^{\varepsilon}(\cdot), \bar{\alpha}^{\varepsilon}(\cdot)\right)$ converges weakly to $(x(\cdot), \bar{\alpha}(\cdot))$, which is the solution of the martingale problem with operator $\overline{\mathcal{L}}$.

The proof is divided into the following steps. First, we prove the tightness of $x^{\varepsilon}(\cdot)$. Once the tightness is verified, we proceed to obtain the convergence by using a martingale problem formulation.

Step (i): Tightness. We first show that a priori bound holds.

Lemma 4.2. Let $x^{\varepsilon}(t)$ denote flow of system corresponding to $\alpha^{\varepsilon}(t)$. Then

$$
\sup _{0 \leq t \leq T} E\left|x^{\varepsilon}(t)\right|^{2}=O(1)
$$

Proof. Recall that

$$
\begin{aligned}
d x^{\varepsilon}(t)= & {\left[r\left(t, \alpha^{\varepsilon}(t)\right) x^{\varepsilon}(t)-\rho\left(t, \alpha^{\varepsilon}(t)\right) x^{\varepsilon}(t)-\rho\left(t, \alpha^{\varepsilon}(t)\right)(\lambda-z) \bar{H}\left(t, \bar{\alpha}^{\varepsilon}(t)\right)\right] d t } \\
& +\sum_{i=1}^{d} \sqrt{\left(\sum_{n=1}^{d_{1}} u_{n}^{\varepsilon, *}\left(t, x^{\varepsilon}(t), \alpha^{\varepsilon}(t)\right) \sigma_{n i}\left(t, \alpha^{\varepsilon}(t)\right)\right)^{2}} d w_{i}(t) \\
x^{\varepsilon}(0)= & x .
\end{aligned}
$$

So,

$$
\begin{aligned}
E\left|x^{\varepsilon}(t)\right|^{2} \leq & \left.K|x|^{2}+E \mid \int_{0}^{t}\left(r\left(\nu, \alpha^{\varepsilon}(\nu)\right)+\rho\left(\nu, \alpha^{\varepsilon}(\nu)\right)\right) x^{\varepsilon}(\nu)\right)\left.d \nu\right|^{2} \\
& +K E \int_{0}^{t}\left(\sum_{n=1}^{d_{1}} u_{n}^{\varepsilon, *}\left(\nu, x^{\varepsilon}(\nu), \alpha^{\varepsilon}(\nu)\right) \sigma_{n i}\left(\nu, \alpha^{\varepsilon}(\nu)\right)\right)^{2} d \nu \\
\leq & K+K E \int_{0}^{t}\left|x^{\varepsilon}(\nu)\right|^{2} d \nu .
\end{aligned}
$$

Here, recall that $\sigma\left(t, \alpha^{\varepsilon}(t)\right)=\left(\sigma_{n i}\left(t, \alpha^{\varepsilon}(t)\right)\right) \in \mathbb{R}^{d_{1} \times d}$ and note that $u_{n}^{\varepsilon, *}$ is the $n$th component of the $d_{1}$ dimensional variable. Using properties of stochastic integrals, Hölder inequality, and boundedness of $r(\cdot), B(\cdot), \sigma(\cdot)$, by Gronwall's inequality, we obtain the second moment bound of $x^{\varepsilon}(t)$ as desired. 
Lemma 4.3. $\left\{x^{\varepsilon}(\cdot)\right\}$ is tight in $D([0, T]: \mathbb{R})$, the space of real-valued functions defined on $[0, T]$ that are right-continuous, and have left limits endowed with the Skorohod topology.

Proof. Denote by $\mathcal{F}_{t}^{\varepsilon}$ the $\sigma$-algebra generated $\left\{w(s), \alpha^{\varepsilon}(s): s \leq t\right\}$ and by $E_{t}^{\varepsilon}$ the conditional expectation w.r.t. $\mathcal{F}_{t}^{\varepsilon}$. For any $T<\infty$, any $0 \leq t \leq T$, any $s>0$, and any $\delta>0$ with $0<s \leq \delta$, by properties of stochastic integral and boundedness of coefficients,

$$
\begin{aligned}
& E_{t}^{\varepsilon}\left|x^{\varepsilon}(t+s)-x^{\varepsilon}(t)\right|^{2} \\
\leq & K E_{t}^{\varepsilon} \int_{t}^{t+s}\left|\left(r\left(\nu, \alpha^{\varepsilon}(\nu)\right)+\rho\left(\nu, \alpha^{\varepsilon}(\nu)\right)\right) x^{\varepsilon}(\nu)\right|^{2} d \nu \\
& +K E_{t}^{\varepsilon} \int_{t}^{t+s}\left(\sum_{n=1}^{d_{1}} u_{n}^{\varepsilon, *}\left(\nu, x^{\varepsilon}(\nu), \alpha^{\varepsilon}(\nu)\right) \sigma_{n i}\left(\nu, \alpha^{\varepsilon}(\nu)\right)\right)^{2} d \nu \\
\leq & K s+K E_{t}^{\varepsilon} \int_{t}^{t+s}\left|x^{\varepsilon}(\nu)\right|^{2} d \nu .
\end{aligned}
$$

Thus we have

$$
\lim _{\delta \rightarrow 0} \limsup _{\varepsilon \rightarrow 0} \sup _{0 \leq s \leq \delta}\left\{E\left[E_{t}^{\varepsilon}\left|x^{\varepsilon}(t+s)-x^{\varepsilon}(t)\right|^{2}\right]\right\}=0
$$

Then the tightness criterion [4, Theorem 3] yields that process $x^{\varepsilon}(\cdot)$ is tight. Here and in the following part, $K$ is a generic constant which takes different values in different context.

Step (ii): Using the techniques given in [14, Lemma 7.18], it can be shown that the martingale problem with operator $\overline{\mathcal{L}}$ has a unique solution for each initial condition.

Step (iii): To complete the proof, we characterize the limit process. Since $\left(x^{\varepsilon}(\cdot), \bar{\alpha}^{\varepsilon}(\cdot)\right)$ is tight, we can extract a weakly convergent subsequence. For notional simplicity, we still denote the subsequence by $\left(x^{\varepsilon}(\cdot), \bar{\alpha}^{\varepsilon}(\cdot)\right)$ with limit $(x(\cdot), \bar{\alpha}(\cdot))$. By Skorohod representation with no change of notation, we may assume $\left(x^{\varepsilon}(\cdot), \bar{\alpha}^{\varepsilon}(\cdot)\right)$ converges to $(x(\cdot), \bar{\alpha}(\cdot))$ w.p.1. We next show that the limit $(x(\cdot), \bar{\alpha}(\cdot))$ is a solution of the martingale problem with operator $\overline{\mathcal{L}}$ defined by 4.4 .

Lemma 4.4. The process $x(\cdot)$ is the solution of the martingale problem with the operator $\overline{\mathcal{L}}$. 
Proof. To obtain the desirable result, we need to show

$$
f(x(t), t, \bar{\alpha}(t))-f(x, 0, \alpha)-\int_{0}^{t} \overline{\mathcal{L}} f(x(\nu), \nu, \bar{\alpha}(\nu)) d \nu \text { is a martingale }
$$

for $i \in \mathcal{M}, f(\cdot, i) \in C_{0}^{2,1}\left([0, T], \mathbb{R}^{r}\right)$. This can be done by showing that for any integer $n>0$, any bounded and measurable function $h_{p}(\cdot, \cdot)$ with $p \leq n$, and any $t, s, t_{p}>0$ with $t_{p} \leq t<t+s \leq T$,

$$
\begin{aligned}
E \prod_{p=1}^{n} h_{p}\left(x^{\varepsilon}\left(t_{p}\right), \bar{\alpha}^{\varepsilon}\left(t_{p}\right)\right)[f(x(t+s), t+s, & \bar{\alpha}(t+s))-f(x(t), t, \bar{\alpha}(t)) \\
& \left.-\int_{t}^{t+s} \overline{\mathcal{L}} f(x(\nu), \nu, \bar{\alpha}(\nu)) d \nu\right]=0 .
\end{aligned}
$$

We further deduce that

$$
\begin{aligned}
& \lim _{\varepsilon \rightarrow 0} E \prod_{p=1}^{n} h_{p}\left(x^{\varepsilon}\left(t_{p}\right), \bar{\alpha}^{\varepsilon}\left(t_{p}\right)\right) \\
& \cdot\left(f\left(x^{\varepsilon}(t+s), t+s, \bar{\alpha}^{\varepsilon}(t+s)\right)-f\left(x^{\varepsilon}(t), t, \bar{\alpha}^{\varepsilon}(t)\right)\right) \\
=E \prod_{p=1}^{n} h_{p}\left(x\left(t_{p}\right), \bar{\alpha}\left(t_{p}\right)\right) & \cdot(f(x(t+s), t+s, \bar{\alpha}(t+s))-f(x(t), t, \bar{\alpha}(t))) .
\end{aligned}
$$

Moreover,

$$
\begin{aligned}
& \lim _{\varepsilon \rightarrow 0} E \prod_{p=1}^{n} h_{p}\left(x^{\varepsilon}\left(t_{p}\right), \bar{\alpha}^{\varepsilon}\left(t_{p}\right)\right)\left[\int_{t}^{t+s} \frac{\partial f\left(x^{\varepsilon}(\nu), \nu, \bar{\alpha}^{\varepsilon}(\nu)\right)}{\partial \nu} d \nu\right] \\
= & E \prod_{p=1}^{n} h_{p}\left(x\left(t_{p}\right), \bar{\alpha}\left(t_{p}\right)\right)\left[\int_{t}^{t+s} \frac{\partial f(x(\nu), \nu, \bar{\alpha}(\nu))}{\partial \nu} d \nu\right]
\end{aligned}
$$

by the weak convergence of $\left(x^{\varepsilon}(\cdot), \bar{\alpha}^{\varepsilon}(\cdot)\right)$ and the Skorohod representation.

For any $f(\cdot)$ chosen above, define

$$
\widehat{f}\left(x^{\varepsilon}(t), t, \alpha^{\varepsilon}(t)\right)=\sum_{i=1}^{l} f\left(x^{\varepsilon}(t), t, i\right) I_{\left\{\alpha^{\varepsilon}(t) \in \mathcal{M}_{i}\right\}}
$$

since $\left(x^{\varepsilon}(t), \alpha^{\varepsilon}(t)\right)$ is a Markov process, we have

$$
\widehat{f}\left(x^{\varepsilon}(t), t, \alpha^{\varepsilon}(t)\right)-\widehat{f}(x, 0, \alpha)-\int_{0}^{t} \mathcal{L}^{\varepsilon} \widehat{f}\left(x^{\varepsilon}(\nu), \nu, \alpha^{\varepsilon}(\nu)\right) d \nu
$$


is a martingale. Consequently,

$$
\begin{aligned}
E \prod_{p=1}^{n} h_{p}\left(x^{\varepsilon}\left(t_{p}\right), \bar{\alpha}^{\varepsilon}\left(t_{p}\right)\right)\left(\widehat { f } \left(x^{\varepsilon}(t+s), t+\right.\right. & \left.s, \alpha^{\varepsilon}(t+s)\right)-\widehat{f}\left(x^{\varepsilon}(t), t, \alpha^{\varepsilon}(t)\right) \\
& \left.-\int_{t}^{t+s} \mathcal{L}^{\varepsilon} \widehat{f}\left(x^{\varepsilon}(\nu), \nu, \alpha^{\varepsilon}(\nu)\right) d \nu\right)=0 .
\end{aligned}
$$

Note that $\widehat{f}\left(x^{\varepsilon}(t), t, \alpha^{\varepsilon}(t)\right)=f\left(x^{\varepsilon}(t), t, \bar{\alpha}^{\varepsilon}(t)\right)$.

Next we need to show that

$$
\begin{gathered}
\lim _{\varepsilon \rightarrow 0} E \prod_{p=1}^{n} h_{p}\left(x^{\varepsilon}\left(t_{p}\right), \bar{\alpha}^{\varepsilon}\left(t_{p}\right)\right) \int_{t}^{t+s} \mathcal{L}^{\varepsilon} \widehat{f}\left(x^{\varepsilon}(\nu), \nu, \alpha^{\varepsilon}(\nu)\right) d \nu \\
=E \prod_{p=1}^{n} h_{p}\left(x\left(t_{p}\right), \bar{\alpha}\left(t_{p}\right)\right) \int_{t}^{t+s} \overline{\mathcal{L}} f(x(\nu), \nu, \bar{\alpha}(\nu)) d \nu
\end{gathered}
$$

Note that we can rewrite $E \prod_{p=1}^{n} h_{p}\left(x^{\varepsilon}\left(t_{p}\right), \bar{\alpha}^{\varepsilon}\left(t_{p}\right)\right) \int_{t}^{t+s} \mathcal{L}^{\varepsilon} \widehat{f}\left(x^{\varepsilon}(\nu), \nu, \alpha^{\varepsilon}(\nu)\right) d \nu$ as

$$
\begin{aligned}
& E \prod_{p=1}^{n} h_{p}\left(x^{\varepsilon}\left(t_{p}\right), \bar{\alpha}^{\varepsilon}\left(t_{p}\right)\right)\left[\int_{t}^{t+s} \sum_{k=1}^{l} \sum_{j=1}^{m_{k}} Q^{\varepsilon} \widehat{f}\left(x^{\varepsilon}(\nu), \nu, \cdot\right)\left(s_{k j}\right) I_{\left\{\alpha^{\varepsilon}(\nu)=s_{k j}\right\}} d \nu\right. \\
& +\int_{t}^{t+s} \sum_{k=1}^{l} \sum_{j=1}^{m_{k}} \frac{\partial \widehat{f}\left(x^{\varepsilon}(\nu), \nu, s_{k j}\right)}{\partial x} I_{\left\{\alpha^{\varepsilon}(\nu)=s_{k j}\right\}}\left[r\left(\nu, s_{k j}\right) x^{\varepsilon}(\nu)+B\left(\nu, s_{k j}\right) u(\nu)\right] d \nu \\
& \left.+\int_{t}^{t+s} \frac{1}{2} \sum_{k=1}^{l} \sum_{j=1}^{m_{k}}\left[u^{\prime}(\nu) \sigma\left(\nu, s_{k j}\right) \sigma^{\prime}\left(\nu, s_{k j}\right) u(\nu)\right] \frac{\partial^{2} \widehat{f}\left(x^{\varepsilon}(\nu), \nu, s_{k j}\right)}{\partial x^{2}} I_{\left\{\alpha^{\varepsilon}(\nu)=s_{k j}\right\}}\right] d \nu .
\end{aligned}
$$

Since $\widetilde{Q}^{k} \mathbb{1}_{m_{k}}=0$, we have

$$
Q^{\varepsilon} \widehat{f}\left(x^{\varepsilon}(t), t, \cdot\right)\left(s_{k j}\right)=\widehat{Q} \widehat{f}\left(x^{\varepsilon}(t), t, \cdot\right)\left(s_{k j}\right) .
$$

We decompose

$$
E \prod_{p=1}^{n} h_{p}\left(x^{\varepsilon}\left(t_{p}\right), \bar{\alpha}^{\varepsilon}\left(t_{p}\right)\right) \int_{t}^{t+s} \mathcal{L}^{\varepsilon} \widehat{f}\left(x^{\varepsilon}(\nu), \nu, \alpha^{\varepsilon}(\nu)\right) d \nu
$$


as $H_{1}^{\varepsilon}(t+s, t)+H_{2}^{\varepsilon}(t+s, t)$. In which

$$
\begin{aligned}
& H_{1}^{\varepsilon}(t+s, t)=E \prod_{p=1}^{n} h_{p}\left(x^{\varepsilon}\left(t_{p}\right), \bar{\alpha}^{\varepsilon}\left(t_{p}\right)\right) \\
& \cdot\left[\sum_{k=1}^{l} \sum_{j=1}^{m_{k}} \int_{t}^{t+s} \mu_{j}^{k} \frac{\partial \widehat{f}\left(x^{\varepsilon}(\nu), \nu, s_{k j}\right)}{\partial x} I_{\left\{\bar{\alpha}^{\varepsilon}(\nu)=k\right\}}\left[r\left(\nu, s_{k j}\right) x^{\varepsilon}(\nu)+B\left(\nu, s_{k j}\right) u(\nu)\right] d \nu\right. \\
& +\frac{1}{2} \sum_{k=1}^{l} \sum_{j=1}^{m_{k}} \int_{t}^{t+s} \mu_{j}^{k}\left[u^{\prime}(\nu) \sigma\left(\nu, s_{k j}\right) \sigma^{\prime}\left(\nu, s_{k j}\right) u(\nu)\right] \frac{\partial^{2} \widehat{f}\left(x^{\varepsilon}(\nu), \nu, s_{k j}\right)}{\partial x^{2}} I_{\left\{\bar{\alpha}^{\varepsilon}(\nu)=k\right\}} d \nu \\
& \left.+\sum_{k=1}^{l} \sum_{j=1}^{m_{k}} \int_{t}^{t+s} \mu_{j}^{k} \widehat{Q} \widehat{f}\left(x^{\varepsilon}(\nu), \nu, \cdot\right)\left(s_{k j}\right) I_{\left\{\bar{\alpha}^{\varepsilon}(\nu)=k\right\}} d \nu\right]
\end{aligned}
$$

and $H_{2}^{\varepsilon}(t+s, t)$ can be represented as

$$
\begin{aligned}
H_{2}^{\varepsilon}(t & +s, t)=E \prod_{p=1}^{n} h_{p}\left(x^{\varepsilon}\left(t_{p}\right), \bar{\alpha}^{\varepsilon}\left(t_{p}\right)\right) \\
& \cdot\left(\sum_{k=1}^{l} \sum_{j=1}^{m_{k}} \int_{t}^{t+s}\left(I_{\left\{\alpha^{\varepsilon}(\nu)=s_{k j}\right\}}-\mu_{j}^{k} I_{\left\{\bar{\alpha}^{\varepsilon}(\nu)=k\right\}}\right) \frac{\partial \widehat{f}\left(x^{\varepsilon}(\nu), \nu, s_{k j}\right)}{\partial x} \cdot\left[r\left(\nu, s_{k j}\right) x^{\varepsilon}(\nu)+B\left(\nu, s_{k j}\right) u(\nu)\right] d \nu\right. \\
& +\sum_{k=1}^{l} \sum_{j=1}^{m_{k}} \int_{t}^{t+s}\left(I_{\left\{\alpha^{\varepsilon}(\nu)=s_{k j}\right\}}-\mu_{j}^{k} I_{\left\{\bar{\alpha}^{\varepsilon}(\nu)=k\right\}}\right) \widehat{Q} \widehat{f}\left(x^{\varepsilon}(\nu), \nu, \cdot\right)\left(s_{k j}\right) d \nu \\
& +\frac{1}{2} \sum_{k=1}^{l} \sum_{j=1}^{m_{k}} \int_{t}^{t+s}\left(I_{\left\{\alpha^{\varepsilon}(\nu)=s_{k j}\right\}}-\mu_{j}^{k} I_{\left\{\bar{\alpha}^{\varepsilon}(\nu)=k\right\}}\right) \\
& \left.\cdot\left[u^{\prime}(\nu) \sigma\left(\nu, s_{k j}\right) \sigma^{\prime}\left(\nu, s_{k j}\right) u(\nu)\right] \frac{\partial^{2} \widehat{f}\left(x^{\varepsilon}(\nu), \nu, s_{k j}\right)}{\partial x^{2}} d \nu\right) .
\end{aligned}
$$

By virtue of Lemma 5.1, [14, Theorem7.14], Cauchy-Schwartz inequality, boundedness of $h_{p}(\cdot), r(\cdot)$ and $B(\cdot)$, for each $k=1,2, \ldots, l ; j=1,2, \ldots, m_{k}$, as $\varepsilon \rightarrow 0$

$$
\begin{aligned}
E \mid \prod_{p=1}^{n} h_{p}\left(x^{\varepsilon}\left(t_{p}\right), \bar{\alpha}^{\varepsilon}\left(t_{p}\right)\right) \int_{t}^{t+s}\left(I_{\left\{\alpha^{\varepsilon}(\nu)=s_{k j}\right\}}-\mu_{j}^{k} I_{\left\{\bar{\alpha}^{\varepsilon}(\nu)=k\right\}}\right) \frac{\partial \widehat{f}\left(x^{\varepsilon}(\nu), \nu, s_{k j}\right)}{\partial x} \\
\left.\cdot\left[r\left(\nu, s_{k j}\right) x^{\varepsilon}(\nu)+B\left(\nu, s_{k j}\right) u(\nu)\right] d \nu\right|^{2} \rightarrow 0 .
\end{aligned}
$$


Similarly as $\varepsilon \rightarrow 0$,

$$
\begin{aligned}
E \mid \prod_{p=1}^{n} h_{p}\left(x^{\varepsilon}\left(t_{p}\right), \bar{\alpha}^{\varepsilon}\left(t_{p}\right)\right) \int_{t}^{t+s} & \left(I_{\left\{\alpha^{\varepsilon}(\nu)=s_{k j}\right\}}-\mu_{j}^{k} I_{\left\{\bar{\alpha}^{\varepsilon}(\nu)=k\right\}}\right) \\
\cdot & {\left[u^{\prime}(\nu) \sigma\left(\nu, s_{k j}\right) \sigma^{\prime}\left(\nu, s_{k j}\right) u(\nu)\right] } \\
\cdot & \left.\frac{\partial^{2} \widehat{f}\left(x^{\varepsilon}(\nu), \nu, s_{k j}\right)}{\partial x^{2}} d \nu\right|^{2} \rightarrow 0,
\end{aligned}
$$

and

$$
\begin{array}{r}
E \mid \prod_{p=1}^{n} h_{p}\left(x^{\varepsilon}\left(t_{p}\right), \bar{\alpha}^{\varepsilon}\left(t_{p}\right)\right) \int_{t}^{t+s}\left(I_{\left\{\alpha^{\varepsilon}(\nu)=s_{k j}\right\}}-\mu_{j}^{k} I_{\left\{\bar{\alpha}^{\varepsilon}(\nu)=k\right\}}\right) \\
\left.\cdot \widehat{Q} \widehat{f}\left(x^{\varepsilon}(\nu), \nu, \cdot\right)\left(s_{k j}\right) d \nu\right|^{2} \rightarrow 0 .
\end{array}
$$

Therefore, $H_{2}^{\varepsilon}(t+s, t)$ converges to 0 in probability. On the other hand, we obtain

$$
\begin{aligned}
& E \prod_{p=1}^{n} h_{p}\left(x^{\varepsilon}\left(t_{p}\right), \bar{\alpha}^{\varepsilon}\left(t_{p}\right)\right) \sum_{k=1}^{l} \sum_{j=1}^{m_{k}} \int_{t}^{t+s} \mu_{j}^{k} \frac{\partial \widehat{f}\left(x^{\varepsilon}(\nu), \nu, s_{k j}\right)}{\partial x} \\
& \cdot\left[r\left(\nu, s_{k j}\right) x^{\varepsilon}(\nu)+B\left(\nu, s_{k j}\right) u(\nu)\right] \\
& \cdot I_{\left\{\bar{\alpha}^{\varepsilon}(\nu)=k\right\}} d \nu \\
& \rightarrow \sum_{k=1}^{l} \sum_{j=1}^{m_{k}} E \prod_{p=1}^{n} h_{p}\left(x\left(t_{p}\right), \bar{\alpha}\left(t_{p}\right)\right) \int_{t}^{t+s} \mu_{j}^{k} \frac{\partial f(x(\nu), \nu, \bar{\alpha}(\nu))}{\partial x} \\
& \cdot\left[r\left(\nu, s_{k j}\right) x(\nu)+B\left(\nu, s_{k j}\right) u(\nu)\right] \\
& \cdot I_{\{\bar{\alpha}(\nu)=k\}} d \nu \\
& =\sum_{k=1}^{l} E \prod_{p=1}^{n} h_{p}\left(x\left(t_{p}\right), \bar{\alpha}\left(t_{p}\right)\right) \int_{t}^{t+s} \frac{\partial f(x(\nu), \nu, \bar{\alpha}(\nu))}{\partial x} \\
& \cdot[\bar{r}(\nu, \bar{\alpha}(\nu)) x(\nu)+\bar{B}(\nu, \bar{\alpha}(\nu)) u(\nu))] \\
& \text { - } I_{\{\bar{\alpha}(\nu)=k\}} d \nu \\
& =E \prod_{p=1}^{n} h_{p}\left(x\left(t_{p}\right), \bar{\alpha}\left(t_{p}\right)\right) \int_{t}^{t+s} \frac{\partial f(x(\nu), \nu, \bar{\alpha}(\nu))}{\partial x} \\
& \cdot[\bar{r}(\nu, \bar{\alpha}(\nu)) x(\nu)+\bar{B}(\nu, \bar{\alpha}(\nu)) u(\nu)] d \nu .
\end{aligned}
$$


Similarly,

$(4.8)$

$$
\begin{aligned}
& E \prod_{p=1}^{n} h_{p}\left(x^{\varepsilon}\left(t_{p}\right), \bar{\alpha}^{\varepsilon}\left(t_{p}\right)\right) \sum_{k=1}^{l} \sum_{j=1}^{m_{k}} \int_{t}^{t+s} \mu_{j}^{k}\left[u^{\prime}(\nu) \sigma^{2}\left(\nu, s_{k j}\right) u(\nu)\right] \\
\rightarrow E & \cdot \frac{\partial^{2} \widehat{f}\left(x^{\varepsilon}(\nu), \nu, s_{k j}\right)}{\partial x^{2}} I_{\left\{\bar{\alpha}^{\varepsilon}(\nu)=k\right\}} d \nu \\
& \prod_{p=1}^{n} h_{p}\left(x\left(t_{p}\right), \bar{\alpha}\left(t_{p}\right)\right) \int_{t}^{t+s} \frac{\partial^{2} f(x(\nu), \nu, \bar{\alpha}(\nu))}{\partial x^{2}}\left[u^{\prime}(\nu) \bar{\sigma}^{2}(\nu, \bar{\alpha}(\nu)) u(\nu)\right] d \nu .
\end{aligned}
$$

Note that

$$
\begin{aligned}
& \sum_{k=1}^{l} \sum_{j=1}^{m_{k}} \int_{t}^{t+s} \mu_{j}^{k} I_{\left\{\bar{\alpha}^{\varepsilon}(\nu)=k\right\}} \widehat{Q} \widehat{f}\left(x^{\varepsilon}(\nu), \nu, \cdot\right)\left(s_{k j}\right) d \nu \\
= & \int_{t}^{t+s} \bar{Q} f\left(x^{\varepsilon}(\nu), \nu, \cdot\right)\left(\bar{\alpha}^{\varepsilon}(\nu)\right) d \nu .
\end{aligned}
$$

So as $\varepsilon \rightarrow 0$,

$$
\begin{aligned}
& E \prod_{p=1}^{n} h_{p}\left(x^{\varepsilon}\left(t_{p}\right), \bar{\alpha}^{\varepsilon}\left(t_{p}\right)\right) \int_{t}^{t+s} \bar{Q} f\left(x^{\varepsilon}(\nu), \nu, \cdot\right)\left(\bar{\alpha}^{\varepsilon}(\nu)\right) d \nu \\
\rightarrow & E \prod_{p=1}^{n} h_{p}\left(x\left(t_{p}\right), \bar{\alpha}\left(t_{p}\right)\right) \int_{t}^{t+s} \bar{Q} f(x(\nu), \nu, \cdot)(\bar{\alpha}(\nu)) d \nu .
\end{aligned}
$$

Combining the results from (4.7) to 4.9), we have

$$
\begin{aligned}
& \lim _{\varepsilon \rightarrow 0} E \prod_{p=1}^{n} h_{p}\left(x^{\varepsilon}\left(t_{p}\right), \bar{\alpha}^{\varepsilon}\left(t_{p}\right)\right) \int_{t}^{t+s} \mathcal{L}^{\varepsilon} \widehat{f}\left(x^{\varepsilon}(\nu), \nu, \alpha^{\varepsilon}(\nu)\right) d \nu \\
= & E \prod_{p=1}^{n} h_{p}\left(x\left(t_{p}\right), \bar{\alpha}\left(t_{p}\right)\right) \int_{t}^{t+s} \overline{\mathcal{L}} f(x(\nu), \nu, \bar{\alpha}(\nu)) d \nu
\end{aligned}
$$

Finally, piecing together the results obtained, the proof of the theorem is completed.

To proceed, we can further deduce the following result. The proof is omitted. 
Theorem 4.5. For $k=1,2, \ldots, l$ and $j=1,2, \ldots, m_{k}, P^{\varepsilon}\left(t, s_{k j}\right) \rightarrow \bar{P}(t, k)$ and $H^{\varepsilon}\left(t, s_{k j}\right) \rightarrow \bar{H}(t, k)$ uniformly on $[0, T]$ as $\varepsilon \rightarrow 0$, where $\bar{P}(t, k)$ and $\bar{H}(t, k)$ are the unique solutions of the following differential equations for $k=1,2, \ldots, l$,

$$
\begin{aligned}
\dot{\bar{P}}(t, k) & =(\bar{\rho}(t, k)-2 \bar{r}(t, k)) \bar{P}(t, k)-\overline{Q P}(t, \cdot)(k) \\
\bar{P}(T, k) & =1
\end{aligned}
$$

and

$$
\begin{aligned}
\dot{\bar{H}}(t, k)= & \bar{r}(t, k) \bar{H}(t, k)-\frac{1}{\bar{P}(t, k)} \overline{Q P}(t, \cdot) \bar{H}(t, \cdot)(k) \\
& +\frac{\bar{H}(t, k)}{\bar{P}(t, k)} \overline{Q P}(t, \cdot)(k) \\
\bar{H}(T, k)= & 1 .
\end{aligned}
$$

\section{Near optimality and numerical examples}

This section establishes near optimality of the control obtained from the limit system and presents related numerical results.

\subsection{Near Optimality}

By the convergency of $P^{\varepsilon}\left(t, s_{k j}\right)$ to $\bar{P}(t, k)$ and $H^{\varepsilon}\left(t, s_{k j}\right)$ to $\bar{H}(t, k)$, we have $v^{\varepsilon}\left(t, s_{k j}, x\right) \rightarrow \bar{v}(t, k, x)$ as $\varepsilon \rightarrow 0$, in which $\bar{v}(t, k, x)=\bar{P}(t, k)(x+(\lambda-$ $z) \bar{H}(t, k))^{2}$. Here, $\bar{v}(t, k, x)$ corresponds to the value function of a limit problem. In view of Theorem 4.1, for the limit problem, let $\mathcal{U}$ be the control set $\mathcal{U}=\left\{U=\left(U^{1}, U^{2}, \ldots, U^{l}\right): U^{k}=\left(u^{k 1}, u^{k 2}, \ldots, u^{k m_{k}}\right), u^{k j} \in \mathbb{R}^{d_{1}}\right\}$. Define

$$
\begin{aligned}
\Phi(t, x, k, U) & =\sum_{j=1}^{m_{k}} \mu_{j}^{k} r\left(t, s_{k j}\right) x+\sum_{j=1}^{m_{k}} \mu_{j}^{k} B\left(t, s_{k j}\right) u^{k j}(t) \text { and } \\
\Psi(t, k, U) & =\left(\left(g_{1}(t, k, U)\right), \ldots, g_{d}(t, k, U)\right) \text { with } \\
\Psi_{i}(t, k, U) & =\sqrt{\sum_{j=1}^{m_{k}} \mu_{j}^{k}\left(\sum_{n=1}^{d_{1}} u_{n}^{k j} \sigma_{n i}\left(t, s_{k j}\right)\right)^{2}} .
\end{aligned}
$$

Here, recall that $\sigma\left(t, \alpha^{\varepsilon}(t)\right)=\left(\sigma_{n i}\left(t, s_{k j}\right)\right) \in \mathbb{R}^{d_{1} \times d}$ and note that $u_{n}^{k j}$ is the $n$th component of the $d_{1}$ dimensional variable. The corresponding dynamic 
system is given by

$$
d x(t)=\Phi(t, x(t), \bar{\alpha}(t), U(t)) d t+\sum_{i=1}^{d} \Psi_{i}(t, \bar{\alpha}(t), U(t)) d w_{i}(t)
$$

where $\bar{\alpha}(\cdot) \in\{1,2, \ldots, l\}$ is a Markov chain generated by $\bar{Q}$ with $\bar{\alpha}(0)=\alpha$. It can be shown that the optimal control for this limit problem is

$$
U^{*}(t, x)=\left(U^{1 *}(t, x), U^{2 *}(t, x), \ldots, U^{l *}(t, x)\right)
$$

with

$$
U^{k *}(t, x)=\left(u^{k 1 *}(t, x), u^{k 2 *}(t, x), \ldots, u^{k m_{k} *}(t, x)\right)
$$

and

$$
u^{k j *}(t, x)=-\left(\sigma\left(t, s_{k j}\right) \sigma^{\prime}\left(t, s_{k j}\right)\right)^{-1} B^{\prime}\left(t, s_{k j}\right)[x+(\lambda-z) \bar{H}(t, k)]
$$

Using such controls, we construct

$$
u^{\varepsilon}\left(t, \alpha^{\varepsilon}(t), x\right)=\sum_{k=1}^{l} \sum_{j=1}^{m_{k}} I_{\left\{\alpha^{\varepsilon}(t)=s_{k j}\right\}} u^{k j *}(t, x)
$$

for the original problem. This control can also be written as if

$$
\begin{aligned}
\alpha^{\varepsilon}(t) \in \mathcal{M}_{k}, u^{\varepsilon}\left(t, \alpha^{\varepsilon}(t), x\right)= & -\left(\sigma\left(t, \alpha^{\varepsilon}(t)\right) \sigma^{\prime}\left(t, \alpha^{\varepsilon}(t)\right)\right)^{-1} \\
& \cdot B^{\prime}\left(t, \alpha^{\varepsilon}(t)\right)\left[x+(\lambda-z) \bar{H}\left(t, \bar{\alpha}^{\varepsilon}(t)\right)\right] .
\end{aligned}
$$

It can be shown that our constructed control is nearly optimal. We present the following lemmas first.

Lemma 5.1. For any $k=1,2, \ldots, l, j=1,2, \ldots, m_{k}$, we have the following result hold.

$$
E\left|\int_{0}^{t}\left[I_{\left\{\alpha^{\varepsilon}(\nu)=s_{k j}\right\}}-\mu_{j}^{k} I_{\left\{\bar{\alpha}^{\varepsilon}(\nu)=k\right\}}\right] x^{\varepsilon}(\nu) r\left(\nu, \alpha^{\varepsilon}(\nu)\right) d \nu\right|^{2} \rightarrow 0 \text { as } \varepsilon \rightarrow 0 .
$$

Proof. For $0<\delta<1$ and any $t \in[0, T]$, let $N=\left[t / \varepsilon^{1-\delta}\right]$ partition $[0, t]$ into subintervals of equal length $\varepsilon^{1-\delta}$ and denote the partition boundaries by 
$t_{k}=k \varepsilon^{1-\delta}$ for $0 \leq k \leq N-1$. Define the auxiliary function

$$
\widetilde{F}(\nu)=r\left(\nu, \alpha^{\varepsilon}(\nu)\right) x^{\varepsilon}\left(t_{k}\right), \quad \text { for } u \in\left[t_{k}, t_{k+1}\right] .
$$

Lemma 4.3 shows

$$
E\left|x^{\varepsilon}(t)-x^{\varepsilon}\left(t_{k}\right)\right|^{2}=O\left(\varepsilon^{1-\delta}\right) \rightarrow 0 \text { as } \varepsilon \rightarrow 0 .
$$

for $t \in\left[t_{k}, t_{k+1}\right], 0 \leq k \leq N-1$. Then

$$
\begin{aligned}
& E\left|\int_{0}^{t}\left[I_{\left\{\alpha^{\varepsilon}(\nu)=s_{k j}\right\}}-\mu_{j}^{k} I_{\left\{\bar{\alpha}^{\varepsilon}(\nu)=k\right\}}\right] x^{\varepsilon}(\nu) r\left(\nu, \alpha^{\varepsilon}(\nu)\right) d \nu\right|^{2} \\
\leq & 2 E\left|\int_{0}^{t}\left[I_{\left\{\alpha^{\varepsilon}(\nu)=s_{k j}\right\}}-\mu_{j}^{k} I_{\left\{\bar{\alpha}^{\varepsilon}(\nu)=k\right\}}\right] \widetilde{F}(\nu) d \nu\right|^{2} \\
& +2 E\left|\int_{0}^{t}\left[I_{\left\{\alpha^{\varepsilon}(\nu)=s_{k j}\right\}}-\mu_{j}^{k} I_{\left\{\bar{\alpha}^{\varepsilon}(\nu)=k\right\}}\right]\left(x^{\varepsilon}(\nu) r\left(\nu, \alpha^{\varepsilon}(\nu)\right)-\widetilde{F}(\nu)\right) d \nu\right|^{2} .
\end{aligned}
$$

First, we estimate the last term of (5.4). According to Cauchy-Schwartz inequality, we have

$$
\begin{aligned}
& E\left|\int_{0}^{t}\left[I_{\left\{\alpha^{\varepsilon}(\nu)=s_{k j}\right\}}-\mu_{j}^{k} I_{\left\{\bar{\alpha}^{\varepsilon}(\nu)=k\right\}}\right]\left(x^{\varepsilon}(\nu) r\left(\nu, \alpha^{\varepsilon}(\nu)\right)-\widetilde{F}(\nu)\right) d \nu\right|^{2} \\
\leq & K \int_{0}^{t} E\left|x^{\varepsilon}(\nu) r\left(\nu, \alpha^{\varepsilon}(\nu)\right)-\widetilde{F}(\nu)\right|^{2} d \nu \\
\leq & K \sum_{k=0}^{N-1} \int_{t_{k}}^{t_{k+1}} E\left|x^{\varepsilon}(\nu) r\left(\nu, \alpha^{\varepsilon}(\nu)\right)-r\left(\nu, \alpha^{\varepsilon}(\nu)\right) x^{\varepsilon}\left(t_{k}\right)\right|^{2} d \nu \\
\leq & K O\left(\varepsilon^{1-\delta}\right) \rightarrow 0 \text { as } \varepsilon \rightarrow 0 .
\end{aligned}
$$

For the first term of (5.4), for each $k=1,2, \ldots, l$ and $j=1,2, \ldots, m_{k}$ define

$$
\eta^{\varepsilon}(t)=E\left|\int_{0}^{t}\left[I_{\left\{\alpha^{\varepsilon}(\nu)=s_{k j}\right\}}-\mu_{j}^{k} I_{\left\{\bar{\alpha}^{\varepsilon}(\nu)=k\right\}}\right] \widetilde{F}(\nu) d \nu\right|^{2} .
$$

With the similar idea involved in [14, Lemma 7.14], we get

$$
\sup _{0 \leq t \leq T} \eta^{\varepsilon}(t)=\sup _{0 \leq t \leq T} \int_{0}^{t} O\left(\varepsilon^{1-\delta}\right) d \nu=O\left(\varepsilon^{1-\delta}\right) \rightarrow 0 \text { as } \varepsilon \rightarrow 0 .
$$

Thus, we conclude the proof by combining the above two parts. 
Lemma 5.2. For any $k=1,2, \ldots, l, j=1,2, \ldots, m_{k}$, we have the following result hold.

$$
E\left(I_{\left\{\bar{\alpha}^{\varepsilon}(s)=k\right\}}-I_{\{\bar{\alpha}(s)=k\}}\right)^{2} \rightarrow 0 \text { as } \varepsilon \rightarrow 0 .
$$

Proof. Similar to [14, Thm. 7.30], we can see that $\left(I_{\left\{\bar{\alpha}^{\varepsilon}(\cdot)=1\right\}}, \ldots, I_{\left\{\bar{\alpha}^{\varepsilon}(\cdot)=l\right\}}\right)$ converges weakly to $\left(I_{\{\bar{\alpha}(\cdot)=1\}}, \ldots, I_{\{\bar{\alpha}(\cdot)=l\}}\right)$ in $D[0, T]$ as $\varepsilon \rightarrow 0$. By means of Cramér-Word's device, for each $i \in \mathcal{M}, I_{\left\{\bar{\alpha}^{\varepsilon}(\cdot)=i\right\}}$ converges weakly to $I_{\{\bar{\alpha}(\cdot)=i\}}$. Then with Skorohod representation (with a little bit of abuse of notation), we may assume $I_{\left\{\bar{\alpha}^{\varepsilon}(\cdot)=i\right\}} \rightarrow I_{\{\bar{\alpha}(\cdot)=i\}}$ w.p.1 without change of notation. Now by dominance convergence theorem, we can conclude the proof.

The following result was obtained in [13]. We state the result and omit the proof.

Theorem 5.3. The control $u^{\varepsilon}(t)$ defined in $(5.2)$ is nearly optimal in that

$$
\lim _{\varepsilon \rightarrow 0}\left|J^{\varepsilon}\left(\alpha, x, u^{\varepsilon}(\cdot)\right)-v^{\varepsilon}(\alpha, x)\right|=0 .
$$

Next, we consider the case in which the Markov chain has transient states. We assume

$$
\widetilde{Q}=\left(\begin{array}{cc}
\widetilde{Q}_{r} & 0 \\
\widetilde{Q}_{0} & \widetilde{Q}_{*}
\end{array}\right)
$$

where $\widetilde{Q}_{r}=\operatorname{diag}\left(\widetilde{Q}^{1}, \widetilde{Q}^{2}, \ldots, \widetilde{Q}^{l}\right), \widetilde{Q}_{0}=\left(\widetilde{Q}_{*}^{1}, \ldots, \widetilde{Q}_{*}^{l}\right)$. For each $k=1,2, \ldots, l$, $\widetilde{Q}^{k}$ is a generator with dimension $m_{k} \times m_{k}, \widetilde{Q}_{*} \in \mathbb{R}^{m_{*} \times m_{*}}, \widetilde{Q}_{*}^{k} \in \mathbb{R}^{m_{*} \times m_{k}}$, and $m_{1}+m_{2}+\cdots+m_{*}=m$. The state space of the underlying Markov chain is given by $\mathcal{M}=\mathcal{M}_{1} \cup \mathcal{M}_{2} \cup \cdots \cup \mathcal{M}_{*}=\left\{s_{11}, \ldots, s_{1 m_{1}}, \ldots, s_{l 1} \ldots\right.$, $\left.s_{l m_{l}}, s_{* 1}, \ldots, s_{* m_{*}}\right\}$, where $\mathcal{M}_{*}=\left\{s_{* 1}, s_{* 2}, \ldots, s_{* m_{*}}\right\}$ consists of the transient states. Suppose for $k=1,2, \ldots, l, \widetilde{Q}^{k}$ are irreducible, and $\widetilde{Q}_{*}$ is Hurwitz, i.e., it has eigenvalues with negative real parts. Let

$$
\widehat{Q}=\left(\begin{array}{cc}
\widehat{Q}^{11} & \widehat{Q}^{12} \\
\widehat{Q}^{21} & \widehat{Q}^{22}
\end{array}\right)
$$

where $\widehat{Q}^{11} \in \mathbb{R}^{\left(m-m_{*}\right) \times(m-m *)}, \widehat{Q}^{12} \in \mathbb{R}^{\left(m-m_{*}\right) \times m_{*}}, \widehat{Q}^{21} \in \mathbb{R}^{m_{*} \times\left(m-m_{*}\right)}$, and $\widehat{Q}^{22} \in \mathbb{R}^{m_{*} \times m_{*}}$. We define

$$
\bar{Q}_{*}=\operatorname{diag}\left(\mu^{1}, \ldots, \mu^{l}\right)\left(\widehat{Q}^{11} \tilde{\mathbb{1}}+\widehat{Q}^{12}\left(a_{m_{1}}, a_{m_{2}}, \ldots, a_{m_{l}}\right)\right)
$$


with $\tilde{\mathbb{1}}=\operatorname{diag}\left(\mathbb{1}_{m_{1}}, \ldots, \mathbb{1}_{m_{l}}\right), \mathbb{1}_{m_{j}}=(1, \ldots, 1)^{\prime} \in \mathbb{R}^{m_{j}}$ and, for $k=1, \ldots, l$,

$$
a_{m_{k}}=\left(a_{m_{k}, 1}, \ldots, a_{m_{k}, m_{*}}\right)^{\prime}=-\widetilde{Q}_{*}^{-1} \widetilde{Q}_{*}^{k_{1}} \mathbb{1}_{m_{k}}
$$

Let $\xi$ be a random variable uniformly distributed on $[0,1]$ that is independent of $\alpha^{\varepsilon}(\cdot)$. For each $j=1,2, \ldots, m_{*}$, define an integer-valued random variable $\xi_{j}$ by

$$
\xi_{j}=I_{\left\{0 \leq \xi \leq a_{m_{1}, j}\right\}}+2 I_{\left\{a_{m_{1}, j}<\xi \leq a_{m_{1}, j}+a_{m_{2}, j}\right\}}+\cdots+l I_{\left\{a_{m_{1}, j}+\cdots+a_{m_{l-1}, j}<\xi \leq 1\right\}} .
$$

Now define the aggregated process $\bar{\alpha}^{\varepsilon}(\cdot)$ by

$$
\bar{\alpha}^{\varepsilon}(t)= \begin{cases}k, & \text { if } \alpha^{\varepsilon}(t) \in \mathcal{M}_{k}, \\ \xi_{j}, & \text { if } \alpha^{\varepsilon}(t)=s_{* j} .\end{cases}
$$

Note the state space of $\bar{\alpha}^{\varepsilon}(t)$ is $\overline{\mathcal{M}}=\{1,2, \ldots, l\}$ and $\bar{\alpha}^{\varepsilon}(\cdot) \in D[0, T]$. In addition,

$$
P\left(\bar{\alpha}^{\varepsilon}(t)=i \mid \alpha^{\varepsilon}(t)=s_{* j}\right)=a_{m_{i}, j} .
$$

Then according to [15, Theorem 4.2], $\bar{\alpha}^{\varepsilon}(\cdot)$ converges weakly to $\bar{\alpha}(\cdot)$ where $\bar{\alpha}(\cdot) \in\{1,2, \ldots, l\}$ is the Markov chain generated by $\bar{Q}_{*}$. The following two theorems were also proved in [13].

Theorem 5.4. As $\varepsilon \rightarrow 0$, we have $P^{\varepsilon}\left(s, s_{k j}\right) \rightarrow \bar{P}(s, k)$ and $H^{\varepsilon}\left(s, s_{k j}\right) \rightarrow$ $\bar{H}(s, k), \quad$ for $k=1,2, \ldots, l, \quad j=1,2, \ldots, m_{k}, \quad P^{\varepsilon}\left(s, s_{* j}\right) \rightarrow \bar{P}_{*}(s, j) \quad$ and $H^{\varepsilon}\left(s, s_{* j}\right) \rightarrow \bar{H}_{*}(s, j)$, for $j=1,2, \ldots, m_{*}$ uniformly on $[0, T]$ where

$$
\begin{aligned}
& \bar{P}_{*}(s, j)=a_{m_{1}, j} \bar{P}(s, 1)+\cdots+a_{m_{l}, j} \bar{P}(s, l), \\
& \bar{H}_{*}(s, j)=a_{m_{1}, j} \bar{H}(s, 1)+\cdots+a_{m_{l}, j} \bar{H}(s, l)
\end{aligned}
$$

and $\bar{P}(s, k)$ and $\bar{H}(s, k)$ are the unique solutions to the following equations. For $k=1,2, \ldots, l$,

$$
\begin{aligned}
\dot{\bar{P}}(t, k) & =(\bar{\rho}(t, k)-2 \bar{r}(t, k)) \bar{P}(t, k)-\bar{Q}_{*} \bar{P}(t, \cdot)(k), \\
\bar{P}(T, k) & =1
\end{aligned}
$$


And

$$
\begin{aligned}
\dot{\bar{H}}(t, k)= & \bar{r}(t, k) \bar{H}(t, k)-\frac{1}{\bar{P}(t, k)} \bar{Q}_{*} \bar{P}(t, \cdot) \bar{H}(t, \cdot)(k) \\
& +\frac{\bar{H}(t, k)}{\bar{P}(t, k)} \bar{Q}_{*} \bar{P}(t, \cdot)(k) \\
\bar{H}(T, k)= & 1 .
\end{aligned}
$$

Theorem 5.5. Construct

$$
\begin{aligned}
u^{\varepsilon}\left(t, \alpha^{\varepsilon}(t), x\right)= & \sum_{k=1}^{l} \sum_{j=1}^{m_{k}} I_{\left\{\alpha^{\varepsilon}(t)=s_{k j}\right\}} u^{k j *}(t, x) \\
& +\sum_{j=1}^{m_{*}} I_{\left\{\alpha^{\varepsilon}(t)=s_{* j}\right\}} u^{* j *}(t, x)
\end{aligned}
$$

for the original problem where

$$
u^{* j *}(t, x)=-\left(\sigma\left(t, s_{* j}\right) \sigma^{\prime}\left(t, s_{* j}\right)\right)^{-1} B^{\prime}\left(t, s_{* j}\right)\left[x+(\lambda-z) \bar{H}_{*}(t, j)\right] .
$$

Then control $u^{\varepsilon}\left(t, \alpha^{\varepsilon}(t), x\right)$ defined in (5.8) is nearly optimal. That is,

$$
\lim _{\varepsilon \rightarrow 0}\left|J^{\varepsilon}\left(\alpha, x, u^{\varepsilon}(\cdot)\right)-v^{\varepsilon}(\alpha, x)\right|=0 .
$$

\subsection{Numerical Examples}

In this section, we present a couple of examples to demonstrate the performance of our approximation schemes. First, let us consider that the Markov chain has only recurrent states.

Example 5.6. We consider the networked system in which the Markov chain $\alpha^{\varepsilon}(t) \in \mathcal{M}=\{1,2,3,4\}, t \geq 0$ generated by $Q^{\varepsilon}=\widetilde{Q} / \varepsilon+\widehat{Q}$ with

$$
\begin{aligned}
& \widetilde{Q}=\left(\begin{array}{rrrr}
-1 & 1 & 0 & 0 \\
2 & -2 & 0 & 0 \\
0 & 0 & -1 & 1 \\
0 & 0 & 3 & -3
\end{array}\right), \\
& \widehat{Q}=\left(\begin{array}{rrrr}
-2 & 0 & 1 & 1 \\
1 & -2 & 1 & 0 \\
0 & 1 & -1 & 0 \\
1 & 2 & 0 & -3
\end{array}\right)
\end{aligned}
$$


the condition of coefficients for the flows of our system are as follows: consider the following dynamic system model for $t \in[0,5], x^{\varepsilon}(0)=0, r(t, 1)=.5$, $r(t, 2)=-.1, r(t, 3)=.5, r(t, 4)=-.1, B(t, 1)=1, B(t, 2)=2, B(t, 3)=$ $-1, B(t, 4)=-2, \sigma(t, 1)=\sigma(t, 2)=\sigma(t, 3)=\sigma(t, 4)=1$. In this case, we can classify the Markov chain into two recurrent groups $\mathcal{M}_{1}$ and $\mathcal{M}_{2}$. So the corresponding stationary distribution for $\mathcal{M}_{1}$ is $\mu^{1}=\left\{\frac{2}{3}, \frac{1}{3}\right\}$ and that of $\mathcal{M}_{2}$ is $\mu^{2}=\left\{\frac{3}{4}, \frac{1}{4}\right\}$. We discretize the equations with step size $h=.01$. So, in the corresponding discrete time setting the time horizon is $T_{h}=\frac{5}{h}$. Let $x^{\varepsilon}(t)$ be the optimal trajectory and $\bar{x}(t)$ be the nearly optimal trajectory with control taking as $u^{\varepsilon}(\cdot)$. Sample paths of $\alpha^{\varepsilon}(t)$, trajectories of $\left|x^{\varepsilon}(t)-\bar{x}(t)\right|$ are given in Figure 1 for $\varepsilon=0.1$ and in Figure 2 for $\varepsilon=0.01$. The results below are based on computations using 100 sample paths. Define

$$
\begin{aligned}
\left|P^{\varepsilon}-\bar{P}\right|= & \frac{1}{T_{h}} \sum_{j=1}^{T_{h}}\left(\left|P^{\varepsilon}(j h, 1)-\bar{P}(j h, 1)\right|+\left|P^{\varepsilon}(j h, 2)-\bar{P}(j h, 1)\right|\right. \\
& \left.+\left|P^{\varepsilon}(j h, 3)-\bar{P}(j h, 2)\right|+\left|P^{\varepsilon}(j h, 4)-\bar{P}(j h, 2)\right|\right),
\end{aligned}
$$

and

$$
\left|x^{\varepsilon}-\bar{x}\right|=\frac{1}{T_{h}} \sum_{j=1}^{T_{h}}\left|x^{\varepsilon}(j h)-\bar{x}(j h)\right| .
$$

Then we have the error bounds given in Table 1 for different values of $\varepsilon$.

\begin{tabular}{cccc}
\hline$\varepsilon$ & $\left|P^{\varepsilon}-\bar{P}\right|$ & $\left|x^{\varepsilon}-\bar{x}\right|$ & $\left|J^{\varepsilon}-v^{\varepsilon}\right|$ \\
\hline 0.1 & 1.48 & 0.02 & 0.002 \\
0.01 & 0.52 & 0.016 & 0.001 \\
0.001 & 0.11 & 0.004 & 0.0003 \\
\hline
\end{tabular}

Table 1: Error Bounds for Example 5.6 

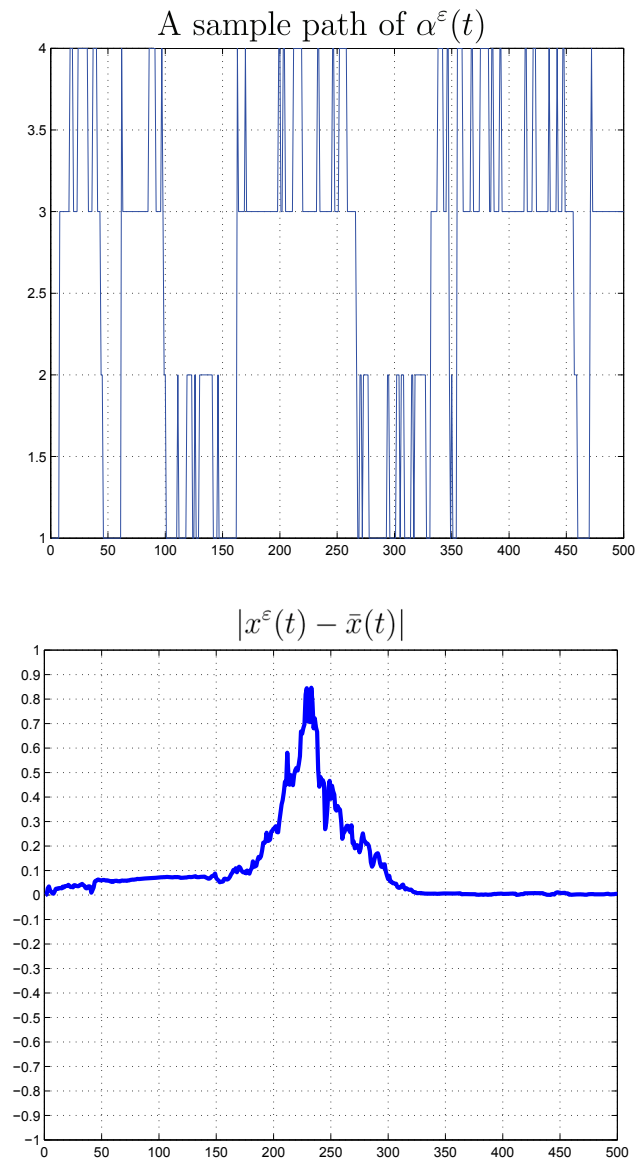

Figure 1: Sample path with $\varepsilon=0.1$ in Example 5.6

Example 5.7. We consider the Markov chain $\alpha^{\varepsilon}(t) \in \mathcal{M}=\{1,2,3,4,5,6\}$ whose generator $Q^{\varepsilon}$ is given by 2.1 with

$$
\begin{aligned}
& \widetilde{Q}=\left(\begin{array}{rrrrrr}
-1 & 1 & 0 & 0 & 0 & 0 \\
2 & -2 & 0 & 0 & 0 & 0 \\
0 & 0 & -1 & 1 & 0 & 0 \\
0 & 0 & 3 & -3 & 0 & 0 \\
0 & 0 & 1 & 1 & -3 & 1 \\
1 & 0 & 0 & 1 & 1 & -3
\end{array}\right), \\
& \widehat{Q}=\left(\begin{array}{rrrrrr}
-1 & 1 & 0 & 0 & 0 & 0 \\
2 & -2 & 0 & 0 & 0 & 0 \\
0 & 0 & -1 & 1 & 0 & 0 \\
0 & 0 & 3 & -3 & 0 & 0 \\
0 & 0 & 1 & 1 & -3 & 1 \\
1 & 0 & 0 & 1 & 1 & -3
\end{array}\right) .
\end{aligned}
$$



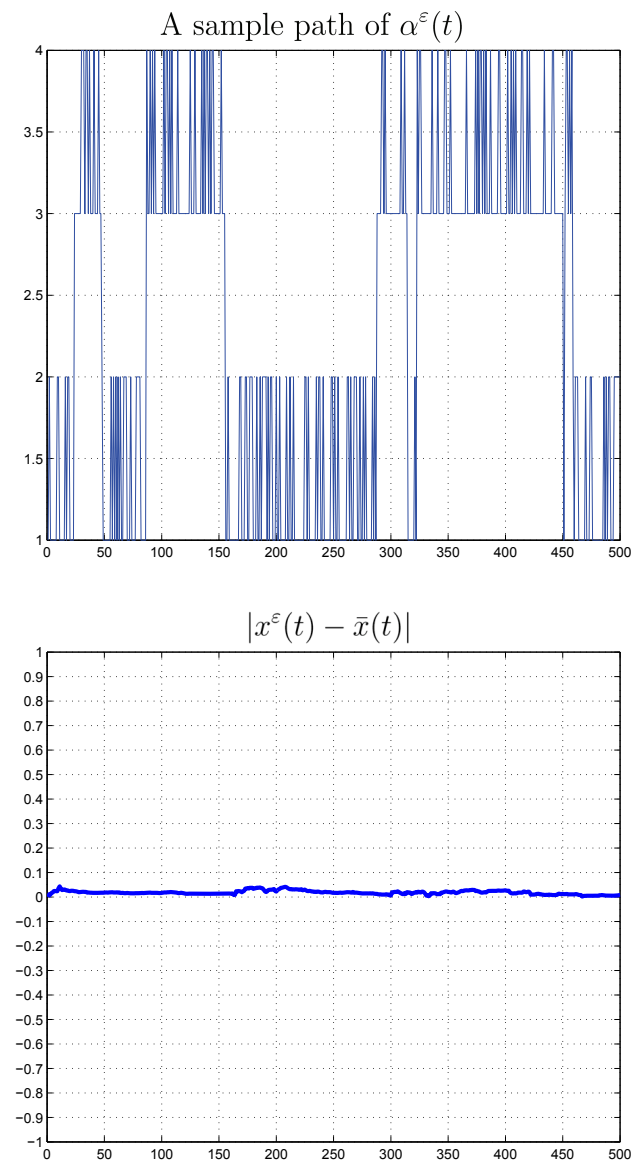

Figure 2: Sample path with $\varepsilon=0.01$ in Example 5.6

We use $x^{\varepsilon}(0)=0, r(t, 1)=.5, r(t, 2)=-.1, \quad r(t, 3)=.5, \quad r(t, 4)=-.1$, $r(t, 5)=.2, r(t, 6)=.4, B(t, 1)=1, B(t, 2)=2, B(t, 3)=-1, B(t, 4)=-2$, $B(t, 5)=1, B(t, 6)=2, \sigma(t, 1)=\sigma(t, 2)=\sigma(t, 3)=\sigma(t, 4)=\sigma(t, 5)=\sigma(t$, $6)=1$. Sample paths of $\alpha^{\varepsilon}(t)$, trajectories of $\left|x^{\varepsilon}(t)-\bar{x}(t)\right|$ are given in Figure 3 for $\varepsilon=.1$ and in Figure 4 for $\varepsilon=.01$. We omit the error bounds here yet the result is similar to Example 5.6.

It can be seen from the two graphs that the smaller the $\varepsilon$ the more rapidly $\alpha^{\varepsilon}(\cdot)$ jumps, which results in better approximations. 

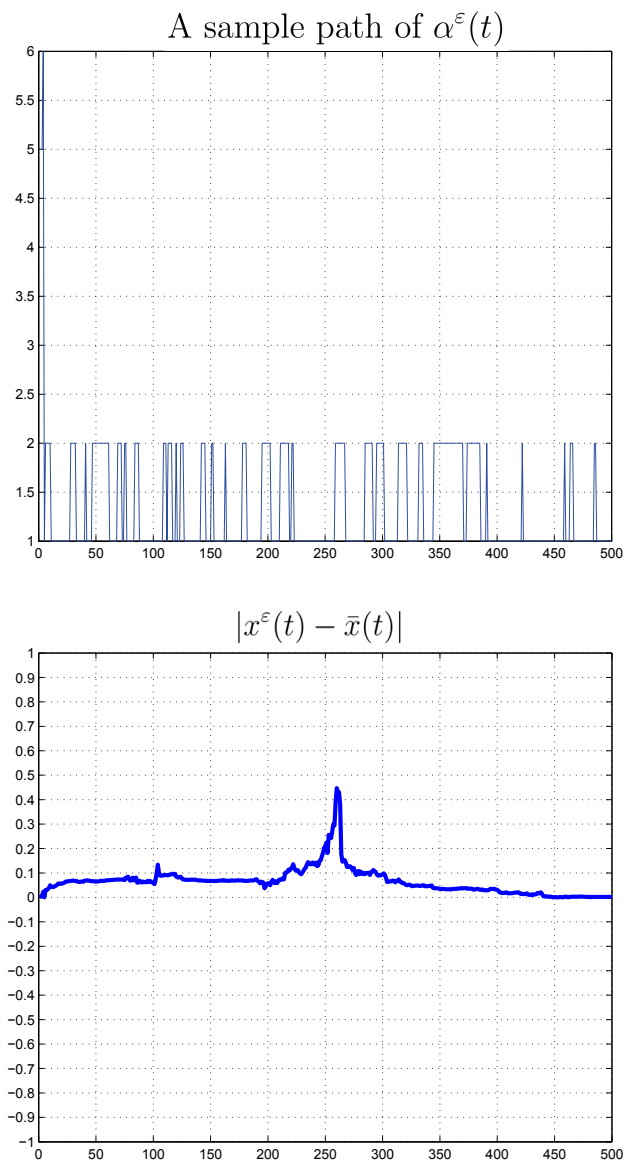

Figure 3: Sample path with $\varepsilon=0.1$ in Example 5.7

\section{Further remarks}

Motivated by platoon control systems, this work establishes a weak convergence result that leads to a limit system of much reduced complexity for mean-variance type of control under randomly regime switching systems. Our methodology uses a two-time-scale formulation to relate the underlying problem with that of the limit problem. Accompanying our recent work [13], this paper also demonstrates the near-optimal controls using numerical examples. Our approach provides a systematic method to reduce the complexity of the underlying system. In lieu of handling large dimensional 

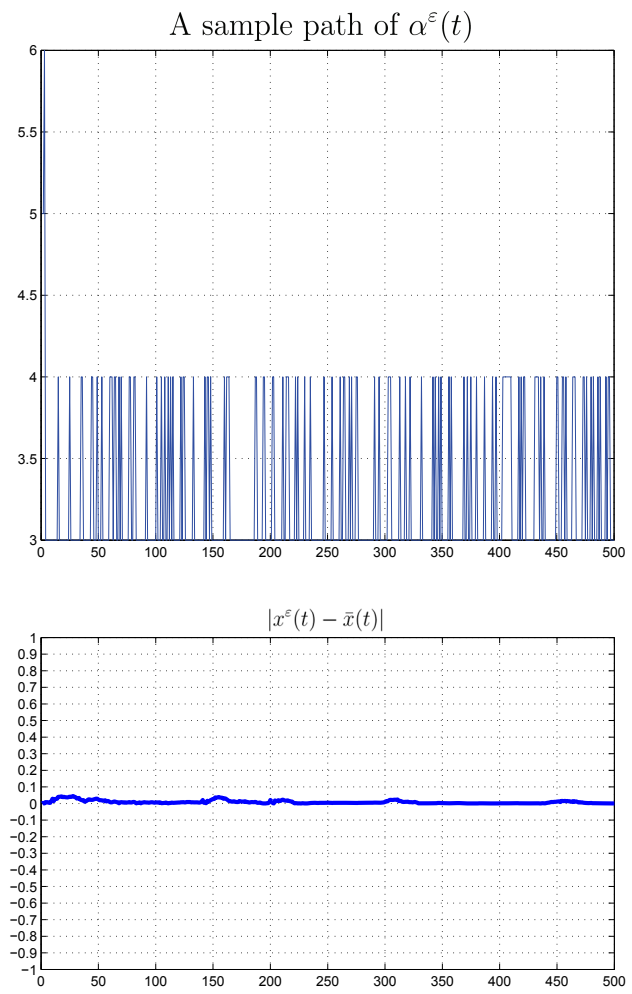

Figure 4: Sample path with $\varepsilon=0.01$ in Example 5.7

systems, we need only solve a reduced set of limit equations that have much smaller dimensions. Future research efforts can be directed to the study of non-definite control problems in the hybrid systems, in which the Markov chain is a hidden process. Then a Wonham filter may be developed. Another direction is to look into the possibility of treating distributed controls with built-in communication complexity measures. All of these deserve more thoughts and further considerations.

\section{References}

[1] S. B. Choi and J. K. Hedrick, Vehicle longitudinal control using an adaptive observer for automated highway systems. In: Proc. of ACC, Seattle, 1995. 
[2] P. J. Courtois, Decomposability: Queueing and Computer System Applications. Academic Press, New York, NY, 1977.

[3] J. K. Hedrick, D. McMahon and D. Swaroop, Vehicle modeling and control for automated highway systems. PATH Research Report, UCBITS-PRR-93-24, 1993.

[4] H. J. Kushner, Approximation and Weak Convergence Methods for Random Processess, with Application to Stochastic Systems Theory. MIT Press, Cambridge, Massachusetts.,1984.

[5] D. Li and W. L. Ng, Optimal dynamic portfolio selection: Multi-period mean-variance formulation. Math. Finance, 10 (2000), 387-406.

[6] C. Y. Liang and H. Peng, String stability analysis of adaptive cruise controlled vehicles. JSME Internat. J. Series C., 43 (2000), 671-677.

[7] H. Markowitz, Portfolio selection. J. Finance, 7 (1952), 77-91.

[8] R. Rajamani, H. S. Tan, B. Law and W. B. Zhang, Demonstration of Integrated Lateral and Longitudinal Control for the Operation of Automated Vehicles in Platoons. IEEE Trans. Control Sys. Tech., 8 (2000), 695-708.

[9] S. P. Sethi and Q. Zhang, Hierarchical Decision Making in Stochastic Manufacturing Systems, Birkhäuser, Boston, MA, 1994.

[10] H. A. Simon and A. Ando, Aggregation of variables in dynamic systems. Econometrica, 29 (1961), 111-138.

[11] D. Swaroop and J. Hedrick, String Stability of Interconnected Systems. IEEE Trans. Automat. Control, 41 (1996), 349-357.

[12] W. S. Wong, Control communication complexity of distributed control systems. SIAM J. Control Optim., 48 (2009), 1722-1742.

[13] Z. Yang, G. Yin, L. Y. Wang and H. Zhang, Near-optimal mean-variance controls under two-time-scale formulations and applications. Stochastics, 85 (2013), 723-741.

[14] G. Yin and Q. Zhang, Continuous-Time Markov Chains and Applications: A Singular Pertubation Approach. New York, Springer-Verlag, 1998.

[15] G. Yin, Q. Zhang and G. Badowski, Asymptotic properties of a singularly perturbed markov chain with inclusion of transient states. The Ann. Appl. Probab., 10 (2000), 549-572. 
[16] Q. Zhang and G. Yin, On nearly optimal controls of hybrid LQG problems. IEEE Trans. Automat. Control., 44 (1999), 2271-2282.

[17] X. Y. Zhou and D. Li, Continuous-time mean-variance portfolio selection: A stochastic LQ framework. Appl. Math. Optim., 42 (2000), 1933.

[18] X. Y. Zhou and G. Yin, Markowitz's mean-variance portfolio selection with regime switching: a continuous time model. SIAM J. Control Optim., 42 (2003), 1466-1482.

Department of Mathematics, University of Wisconsin-Eau Claire

Hibbard Hall 420, Eau Claire, WI 54701, USA

E-mail address: yangzhix@uwec.edu

Department of Mathematics, Wayne State University

Detroit, Michigan 48202, USA

E-mail address: gyin@math.wayne.edu

Department of Electrical and Computer Engineering

WAyne State University

Detroit, MI 48202, USA

E-mail address: lywang@wayne.edu

Department of Computer Science, Wayne State University

Detroit, MI 48202, USA

E-mail address: hongwei@wayne.edu

ReCEIVED June 6, 2012

Accepted October 18, 2012 